Document downloaded from:

http://hdl.handle.net/10251/154496

This paper must be cited as:

Vico Bondía, F.; Greengard, L.; Ferrando Bataller, M.; Antonino Daviu, E. (2019). An Augmented Regularized Combined Source Integral Equation for Nonconforming Meshes. IEEE Transactions on Antennas and Propagation. 67(4):2513-2521. https://doi.org/10.1109/TAP.2019.2891399

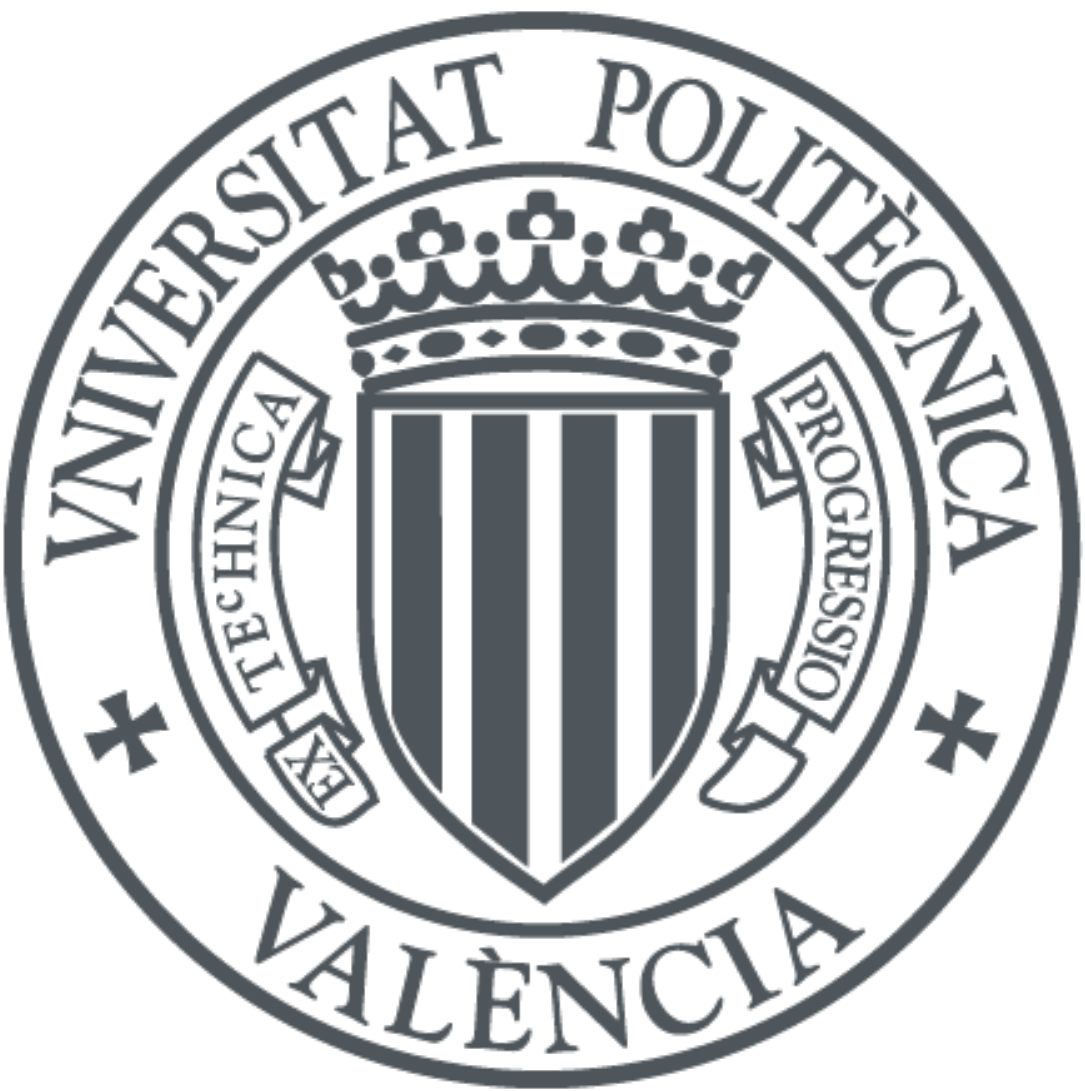

The final publication is available at

https://doi.org/10.1109/TAP.2019.2891399

Copyright Institute of Electrical and Electronics Engineers

Additional Information 


\title{
An Augmented Regularized Combined Source Integral Equation for Nonconforming Meshes
}

\author{
Felipe Vico, Member, IEEE, Leslie Greengard, Member, IEEE Miguel Ferrando-Bataller, Member, IEEE \\ Eva Antonino-Daviu, Member, IEEE,
}

\begin{abstract}
We present a new version of the regularized combined source integral equation (CSIE-AR) for the solution of electromagnetic scattering problems in the presence of perfectly conducting bodies. The integral equation is of the second kind and has no spurious resonances. It is well-conditioned at all frequencies for simply connected geometries. Reconstruction of the magnetic field, however, is subject to catastrophic cancellation due to the need for computing a scalar potential from magnetic currents. Here, we show that by solving an auxiliary (scalar) integral equation, we can avoid this form of low-frequency breakdown. The auxiliary scalar equation is used to solve a Neumanntype boundary value problem using data corresponding to the normal component of the magnetic field. This scalar equation is also of the second kind, non-resonant, and well-conditioned at all frequencies. A principal advantage of our approach, by contrast with hyper-singular EFIE, CFIE or CSIE formulations, is that the standard loop-star and related basis function constructions are not needed, and preconditioners are not required. This permits an easy coupling to fast algorithms such as the fast multipole method (FMM). Furthermore, the formalism is compatible with non-conformal mesh discretization and works well with singular (sharp) boundaries.
\end{abstract}

Index Terms-electromagnetic (EM) scattering, Calderon preconditioning (CP), charge-current formulations, high frequency preconditioning, Maxwell equations.

\section{INTRODUCTION}

I NTEGRAL equation methods are widely used for solving electromagnetic scattering problems since they discretize the boundary alone and permit the imposition of the Sommerfeld radiation condition without the need for artificial boundary conditions. The resulting discretized linear systems, however, are dense so that naive solution methods are impractical for large-scale modeling. At present, the state of the art (in three dimensions) consists of using iterative solution techniques

This work was supported in part by the Spanish Ministry of Science and Innovation (Ministerio de Ciencia e Innovacion) under the projects TEC201678028-C3-3-P and in part by the Office of the Assistant Secretary of Defense for Research and Engineering and AFOSR under NSSEFF Program Award FA9550-10-1-0180.

F. Vico is with the Instituto de Telecomunicaciones y Aplicaciones Multimedia (ITEAM) , Universitat Politècnica de València, e-mail: felipe.vico@gmail.com).

L. Greengard is with the Flatiron Institute, Simons Foundation, 162 Fifth Avenue, NY, NY 10010 and the Courant Institute of Mathematical Sciences, New York University, 251 Mercer Street, New York, NY 10012-1110. email: greengard@cims.nyu.edu.

M. Ferrando-Bataller is with the Instituto de Telecomunicaciones y Aplicaciones Multimedia (ITEAM) Universitat Politècnica de València, e-mail: mferrand@dcom.upv.es).

E. Antonino-Daviu is with the Instituto de Telecomunicaciones y Aplicaciones Multimedia (ITEAM) Universitat Politècnica de València, e-mail evanda@upvnet.upv.es). combined with fast algorithms for computing the necessary matrix-vector products. The net time, using fast multipole acceleration [1], [2], [3], [4] is of the order $O\left(n_{\text {iter }} N \log N\right)$, where $N$ is the system size and $n_{i t e r}$ is the number of iterations required.

With fast algorithms in place, it remains to choose a mathematical and computational framework that leads to a small number of iterations and to robust, accurate and geometrically flexible software. The most widely used techniques, in practice, are the electric field and combined field integral equations (EFIE and CFIE), with RWG basis functions and a conforming mesh model of the scatterer [5], [6], [7]. Both the EFIE and CFIE, however, are subject to numerical difficulties such as ill-conditioning and low frequency or high density mesh breakdown. As a result, a variety of techniques have been developed to mitigate these problems. The use of loopstar basis functions [8], [9], [10], [11], for example, is known to improve performance in terms of accuracy and conditioning in the low frequency regime. The intrinsic ill-conditioning of these equations comes to a large extent from the involvement of hypersingular integral operators, so that preconditioners [12], [13] are important to improve performance, especially when high density meshes are required. A more subtle problem is that the simultaneous use of fast methods in combination with low frequency stabilization techniques, including loopstar basis functions, can produce instabilities [14].

In order to be able to handle large, high-frequency problems, significant effort has been made to construct well-condtioned Fredholm integral equations of the second kind. While we do not seek to review the literature here, these include the use of Calderon identities to precondition the EFIE [15], [16], [13] and the use of regularizing operators to precondition the CFIE [17], [18], [19]. Charge-current formulations have also been developed that lead to well-conditioned formulations, avoiding low frequency breakdown and catastrophic cancellation in the far field [10], [20], [21], [22], [23], [24], [25]. Other solution methods which lead to second-kind and resonance free equations include those based on generalized Debye sources [26], [27] and decoupled potential formulations [28], [29].

In this paper we introduce an augmented regularized combined source integral equation (CSIE-AR) that is of the second kind and yields very modest condition numbers, even in the high frequency regime. By solving an auxiliary scalar equation, we avoid catastrophic cancellation in a manner similar to charge-current schemes. We will show that the proposed formulation yields good accuracy for singular (sharp) geometries 
even with low order discretization and requires a very modest number of triangles to obtain good resolution. Moreover, the scheme works well even when the triangulation is nonconforming. The latter property is extremely useful when meshing complicated structures, since distinct components can be meshed separately and concatenated without geometric complications during the assembly of a full model. Finally, the formulation does not require the use of preconditioners and (unlike hyper-singular formulations) can be combined with FMM acceleration without compromising accuracy.

\section{STANDARD FORMULATIONS}

The problem of electromagnetic scattering from a perfect electric conductor (PEC) $\Omega$ with boundary $\Gamma$ is governed (in the frequency domain) by the Maxwell equations

$$
\nabla \times \boldsymbol{H}^{t o t}=-i \omega \epsilon \boldsymbol{E}^{t o t}, \quad \nabla \times \boldsymbol{E}^{t o t}=i \omega \mu \boldsymbol{H}^{t o t},
$$

subject to the following boundary conditions [30], [31] on the total electric and magnetic fields:

$$
\begin{array}{rlrl}
\boldsymbol{n} \times \boldsymbol{E}^{\mathrm{tot}} & =0, & \boldsymbol{n} \cdot \boldsymbol{H}^{\mathrm{tot}}=0, \\
\boldsymbol{n} \times \boldsymbol{H}^{\mathrm{tot}}=\boldsymbol{J}, & \boldsymbol{n} \cdot \boldsymbol{E}^{\mathrm{tot}}=\frac{\rho}{\epsilon} .
\end{array}
$$

We use the language of scattering theory and write the total field as the sum of a known incoming field and an unknown scattered field:

$$
\boldsymbol{E}^{t o t}=\boldsymbol{E}^{i n}+\boldsymbol{E}^{\text {scat }}, \quad \boldsymbol{H}^{\text {tot }}=\boldsymbol{H}^{i n}+\boldsymbol{H}^{\text {scat }} .
$$

It is well-known that the boundary conditions (2) are redundant and it is sufficient (for example) to enforce the boundary conditions on the tangential components of the electric field alone.

Most integral equation methods begin by representing the scattered field in terms of the vector and scalar potentials $\mathbf{A}, \phi$ in the Lorenz gauge as

$$
\begin{aligned}
\boldsymbol{E}^{\mathrm{scat}} & =i \omega \boldsymbol{A}^{\mathrm{scat}}-\nabla \phi^{\mathrm{scat}}, \\
\boldsymbol{H}^{\mathrm{scat}} & =\frac{1}{\mu} \nabla \times \boldsymbol{A}^{\mathrm{scat}},
\end{aligned}
$$

where

$$
\begin{aligned}
\boldsymbol{A}^{\text {scat }}[\boldsymbol{J}](\boldsymbol{r}) & =\mu S_{k}[\boldsymbol{J}](\boldsymbol{r}) \\
\phi^{\text {scat }}[\rho](\boldsymbol{r}) & =\frac{1}{\epsilon} S_{k}[\rho](\boldsymbol{r})
\end{aligned}
$$

with

$$
\begin{aligned}
S_{k}[\boldsymbol{J}](\boldsymbol{r}) & =\int_{\Gamma} g_{k}\left(\boldsymbol{r}-\boldsymbol{r}^{\prime}\right) \boldsymbol{J}\left(\boldsymbol{r}^{\prime}\right) d A_{\boldsymbol{r}^{\prime}} \\
S_{k}[\rho](\boldsymbol{r}) & =\int_{\Gamma} g_{k}\left(\boldsymbol{r}-\boldsymbol{r}^{\prime}\right) \rho\left(\boldsymbol{r}^{\prime}\right) d A_{\boldsymbol{r}^{\prime}}
\end{aligned}
$$

and

$$
g_{k}(\boldsymbol{r})=\frac{e^{i k|\boldsymbol{r}|}}{4 \pi|\boldsymbol{r}|}
$$

In order for the resulting electromagnetic fields $\boldsymbol{E}^{\text {scat }}, \boldsymbol{H}^{\text {scat }}$, to be Maxwellian, the current and charge ( $\mathbf{J}$ and $\rho$ ) must satisfy the continuity condition

$$
i \omega \rho=\nabla_{\Gamma} \cdot \boldsymbol{J} .
$$

In particular, if this condition is violated, the electric field fails to be divergence-free.

While the standard EFIE is obtained from (4) and $n \times$ $\boldsymbol{E}^{\text {tot }}=0$, the standard magnetic field integral equation (MFIE) is obtained from (5) and $\boldsymbol{n} \times \boldsymbol{H}^{\text {tot }}=\boldsymbol{J}$, and the standard CFIE is obtained as a linear combination of $\boldsymbol{n} \times \boldsymbol{H}^{\text {tot }}=\boldsymbol{J}$ and $-\boldsymbol{n} \times \boldsymbol{n} \times \boldsymbol{E}^{\text {tot }}=0$, it is possible to use additional unknowns (e.g. charge and current) and to make use of the boundary conditions on normal field components in (2) as well. Such formulations are generally referred to as charge-current formulations or, more generally, as augmented formulations [32], [21], [22], [23], [24], [25]. These are discussed briefly in the next section.

\section{A. The Charge Current Integral Equation}

One useful approach to obtaining well-conditioned integral equations is to impose the tangential boundary conditions on the magnetic field and the normal boundary condition on the electric field in (2). The current and charge are then treated as independent unknowns, yielding the system of equations:

$$
\begin{aligned}
\frac{\boldsymbol{J}}{2}-K[\boldsymbol{J}] & =\boldsymbol{n} \times \boldsymbol{H}^{i n} \\
\frac{\rho}{2}+S_{k}^{\prime}[\rho]-i \omega \epsilon \mu \boldsymbol{n} \cdot S_{k}[\boldsymbol{J}] & =\epsilon \boldsymbol{n} \cdot \boldsymbol{E}^{i n}
\end{aligned}
$$

where:

$$
S_{k}^{\prime}[\rho]=\int_{\Gamma} \frac{\partial g_{k}}{\partial n_{\boldsymbol{r}}}\left(\boldsymbol{r}-\boldsymbol{r}^{\prime}\right) \rho\left(\boldsymbol{r}^{\prime}\right) d A_{\boldsymbol{r}^{\prime}}
$$

and:

$$
K[\boldsymbol{J}]=\int_{\Gamma} \boldsymbol{n} \times \nabla \times g_{k}\left(\boldsymbol{r}-\boldsymbol{r}^{\prime}\right) \boldsymbol{J}\left(\boldsymbol{r}^{\prime}\right) d A_{\boldsymbol{r}^{\prime}}
$$

This formulation was introduced to overcome the low frequency breakdown of the EFIE (see [21], [22]) and the inaccuracy of the MFIE (see [20]). It was shown in [24] that, so long as the incoming data was Maxwellian, the solution to (11) satisfies the continuity condition, and that high precision can be achieved in computing the radiated electric field for simply connected geometries, even for subwavelength-size objects. The first of the equations in (11), however, is simply the MFIE and is subject to resonances as the frequency increases.

\section{B. The Non-Resonant Charge Current Integral Equation}

To avoid the spurious resonances of the MFIE, a nonresonant charge-current integral equation was first introduced in [21], [33]. A more recent version, called the combined current charge integral equation (C3IE) was introduced in [23]. The C3IE is obtained from the following four equations:

$$
\begin{gathered}
\frac{\boldsymbol{J}}{2}-K[\boldsymbol{J}]=\boldsymbol{n} \times \boldsymbol{H}^{\text {in }} \\
-i \omega \epsilon \mu \boldsymbol{n} \cdot S_{k}[\boldsymbol{J}]+\frac{\rho}{2}+S_{k}^{\prime}[\rho]=\epsilon \boldsymbol{n} \cdot \boldsymbol{E}^{\text {in }} \\
i \omega \mu \boldsymbol{n} \times S_{k}[\boldsymbol{J}]-\frac{1}{\epsilon} \boldsymbol{n} \times \nabla S_{k}[\rho]=-\boldsymbol{n} \times \boldsymbol{E}^{\text {in }} \\
\nabla \cdot S_{k}[\boldsymbol{J}]-i \omega S_{k}[\rho]=0
\end{gathered}
$$


where (17) comes from the continuity condition (10). Notice that the surface divergence in (10) transforms to a full $3 \mathrm{D}$ divergence in (17) (see [17] page 205).

For this, the tangential boundary conditions on $\boldsymbol{E}$ and $\boldsymbol{H}$ and the normal boundary condition on $\boldsymbol{E}$ are used from (2), supplemented by a less standard condition on the divergence of the electric field:

$$
\left.\nabla \cdot \boldsymbol{E}^{\mathrm{scat}}\right|_{\Gamma}=0 .
$$

Using (14) with (15) and (16) with (17), we get the nonresonant $\mathrm{C} 3 \mathrm{IE}$ :

$$
\begin{aligned}
& \frac{J}{2}-K[\boldsymbol{J}]+\alpha \boldsymbol{n} \times\left\{i \omega \mu \boldsymbol{n} \times S_{k}[\boldsymbol{J}]-\frac{1}{\epsilon} \boldsymbol{n} \times \nabla S_{k}[\rho]\right\} \\
& =\boldsymbol{n} \times \boldsymbol{H}^{\mathrm{in}}-\alpha \boldsymbol{n} \times \boldsymbol{n} \times \boldsymbol{E}^{\mathrm{in}} \\
& \frac{\rho}{2}+S_{k}^{\prime}[\rho]-i \omega \mu \epsilon \boldsymbol{n} \cdot S_{k}[\boldsymbol{J}]+\alpha\left\{\nabla \cdot S_{k}[\boldsymbol{J}]-i \omega S_{k}[\rho]\right\} \\
& =\epsilon \boldsymbol{n} \cdot \boldsymbol{E}^{\text {in }}
\end{aligned}
$$

where $\alpha$ is an arbitrary real positive constant. By construction, the functions $\boldsymbol{J}=\boldsymbol{n} \times\left.\boldsymbol{H}^{\mathrm{tot}}\right|_{\partial D}$ and $\rho=\left.\epsilon \boldsymbol{n} \cdot \boldsymbol{E}^{\mathrm{tot}}\right|_{\partial D}$ form a solution to the system (19). Since equation (19) has been shown to have a unique solution [23], the continuity condition is automatically satisfied. Due to the non-compactness of the operators $\nabla \cdot S_{k}[\boldsymbol{J}]$ and $\boldsymbol{n} \times \nabla S_{k}[\rho]$, the resulting system (19) is not strictly speaking a Fredholm equation of the second kind. Nevertheless, it has similar properties [23]. Unfortunately, while the C3IE solves the low frequency breakdown problem for simply connected geometries and has good conditioning at higher frequencies, it has been shown in [23], [25] that it performs poorly for geometries with sharp features (edges) for the standard discretization techniques used in those references. It has also been shown that it requires substantial mesh refinement to obtain accurate results.

\section{A Combined Source Charge Current Integral EQUATION}

This section contains the main analytic contribution of our paper. We show that a charge-current combined source integral equation has properties similar to the C3IE in terms of conditioning at low and high frequency, but that it also performs well when the scatterer has sharp features. The scheme is based on the following representation, involving both electric current-like and magnetic current-like variables:

$$
\begin{aligned}
\boldsymbol{E}^{\text {scat }} & =\nabla \times S_{k}[\boldsymbol{M}](\boldsymbol{r})+i \alpha \nabla \times \nabla \times S_{k}[\boldsymbol{J}](\boldsymbol{r}) \\
\boldsymbol{H}^{\text {scat }} & =i \omega \epsilon \mu S_{k}[\boldsymbol{M}](\boldsymbol{r})+\nabla \psi(\boldsymbol{r})+i \alpha \nabla \times S_{k}[\boldsymbol{J}](\boldsymbol{r})
\end{aligned}
$$

where the scalar field $\psi(\boldsymbol{r})$ is given by:

$$
\psi(\boldsymbol{r})=\frac{1}{i \omega \mu} \nabla \cdot S_{k}[\boldsymbol{M}](\boldsymbol{r})
$$

we also define an the following operator that will be used shortly:

$$
T[\boldsymbol{J}]=\int_{\Gamma} \boldsymbol{n} \times \nabla \times \nabla \times g_{k}\left(\boldsymbol{r}-\boldsymbol{r}^{\prime}\right) \boldsymbol{J}\left(\boldsymbol{r}^{\prime}\right) d A_{\boldsymbol{r}^{\prime}}
$$

In order to avoid catastrophic cancellation in the evaluation of (21), we introduce an additional scalar unknown $\sigma$ with

$$
\psi(\boldsymbol{x})=S_{k}[\sigma](\boldsymbol{r})+i \beta D_{k}\left[S_{i k}[\sigma]\right](\boldsymbol{r})
$$

where

$$
D_{k}[\sigma](\boldsymbol{r})=\int_{\Gamma} \frac{\partial g_{k}}{\partial n_{\boldsymbol{r}^{\prime}}}\left(\boldsymbol{r}-\boldsymbol{r}^{\prime}\right) \sigma\left(\boldsymbol{r}^{\prime}\right) d A_{\boldsymbol{r}^{\prime}}
$$

The representation used in the equation (23) was introduced by [34] to solve high frequency Helmholtz scalar equations with Neumann boundary conditions. The only goal of that arbitrary choice for the representation of $\psi$ is to obtain a well conditioned second kind integral equation. Finally, we reduce the number of unknowns by defining $\boldsymbol{J}$ in terms of $M$ using the relation

$$
\boldsymbol{J} \equiv \boldsymbol{n} \times S_{i k}^{2}[\boldsymbol{M}]
$$

Here,

$$
S_{i k}[\boldsymbol{M}](\boldsymbol{r}) \equiv \int_{\Gamma} \frac{e^{-{ }_{k}\left|\boldsymbol{r}-\boldsymbol{r}^{\prime}\right|}}{\left|\boldsymbol{r}-\boldsymbol{r}^{\prime}\right|} \boldsymbol{M}\left(\boldsymbol{r}^{\prime}\right) d A_{\boldsymbol{r}^{\prime}}
$$

and $S_{i k}^{2}[\boldsymbol{M}]$ is used to denote the composition of operators:

$$
S_{i k}^{2}[\boldsymbol{M}] \equiv S_{i k}\left[S_{i k}[\boldsymbol{M}]\right]
$$

Note that we have introduced two regularizing operators: $S_{i k}[\sigma]$ which is composed with $D_{k}$ in (23) and the operator $R=\boldsymbol{n} \times S_{i k}^{2}$ which regularizes the operator $T$ (see [17], page 211, and [19]) that appears when applying the boundary condition on the tangent electric field in (20). These will yield a second kind integral equation (as we shall see shortly) with good conditioning even at high frequencies. Other regularizing operators have been shown to be effective previously, such as $R=\boldsymbol{n} \times S_{0}^{2}$ in [17] and $R=\boldsymbol{n} \times S_{i k}$ in [19]. To derive the actual integral equation, we impose boundary conditions on the tangential components of the electric field $\boldsymbol{n} \times \boldsymbol{E}^{\text {scat }}=$ $-\boldsymbol{n} \times \boldsymbol{E}^{\mathrm{in}}$ and on the normal component of the magnetic field $\boldsymbol{n} \cdot \boldsymbol{H}^{\text {scat }}=-\boldsymbol{n} \cdot \boldsymbol{H}^{\text {in }}$.

$$
\begin{array}{r}
\frac{\boldsymbol{M}}{2}+K[\boldsymbol{M}]+i \alpha T\left[\boldsymbol{n} \times S_{i k}^{2}[\boldsymbol{M}]\right]= \\
=-\boldsymbol{n} \times \boldsymbol{E}^{\mathrm{in}} \\
-\frac{\sigma}{2}+S_{k}^{\prime}[\sigma]+i \beta D_{k}^{\prime}\left[S_{i k}[\sigma]\right]=b
\end{array}
$$

where the operator $D_{k}^{\prime}$ is defined as:

$$
D_{k}^{\prime}[\rho]=\frac{\partial}{\partial n_{\boldsymbol{r}}} \int_{\Gamma} \frac{\partial g_{k}}{\partial n_{\boldsymbol{r}^{\prime}}}\left(\boldsymbol{r}-\boldsymbol{r}^{\prime}\right) \rho\left(\boldsymbol{r}^{\prime}\right) d A_{\boldsymbol{r}^{\prime}} .
$$

and the right hand side $b$ in (28) is defined by:

$$
\begin{aligned}
b= & -\boldsymbol{n} \cdot \boldsymbol{H}^{\mathrm{in}}-i \omega \epsilon \mu \boldsymbol{n} \cdot S_{k}[\boldsymbol{M}]- \\
& -i \alpha \boldsymbol{n} \cdot \nabla \times S_{k}\left[\boldsymbol{n} \times S_{i k}^{2}[\boldsymbol{M}]\right]
\end{aligned}
$$

Note that the two integral equations are decoupled and that the first (vector) equation can be solved independently of the second (scalar) equation. The scalar equation for $\sigma$ can then be solved once $M$ and, therefore, the righ-hand side $b$ are known. Note also that, if one is interested only in the scattered electric field, the second auxiliary equation is not needed. Finally, note that the electromagnetic field will be Maxwellian if and only if the scalar function $\sigma$ satisfies the condition (21). As for the C3IE, this can be shown to follow from the uniqueness of solutions to (28) and the fact that the incoming data is Maxwellian.

In practice, the coupling coefficients $\alpha$ and $\beta$ can be optimized to improve the condition number of the resulting 
system. In general, the optimum values will depend on the frequency and geometry. We propose the following heuristic: $\alpha=\max (1, k)$ and $\beta=1$ (see [35], [36]).

\section{High Frequency Asymptotic AnAlysis}

It is informative to investigate the spectral properties of the proposed integral equation (CSIE-AR) for a spherical scatterer. In that case, all of the operators appearing in (19) or (28) can be diagonalized using spherical harmonics $Y_{n m}$ and vector spherical harmonics $\mathbf{X}_{n m}:=\boldsymbol{n} \times \mathbf{U}_{n m}$ (see [26], [36], [37]). This permits us to compute the condition numbers and spectra analytically and compare various formulations.

Figure 1 compares the condition number of the proposed formulation with other known non-resonant formulations. Both CSIE-AR variants show very good performance.

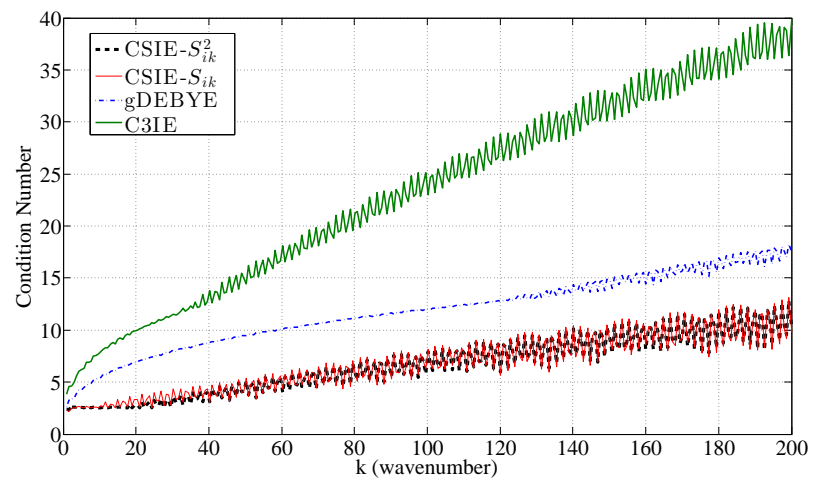

Fig. 1. Condition number of the CSIE-AR using either $S_{i k}^{2}$ or $S_{i k}$ as the regularizer, the generalized Debye method [26] and the CSIE method as a function of frequency.

Figure 2 compares the spectrum at $k=10$ of the CSIE-AR for the proposed regularizing operator $\boldsymbol{n} \times S_{i k}^{2}$, the regularizing operator $\boldsymbol{n} \times S_{i k}$, and the C3IE. As we can see, there is a unique limit point for $C S I E-S_{i k}^{2}$, which is a consequence of the fact that is is a Fredholm integral equation of the second kind. Using the regularizing operator $\boldsymbol{n} \times S_{i k}$ or the C3IE, we find two and three limit points, respectively. In all three cases, the spectra are well-separated from zero and have modest spread.

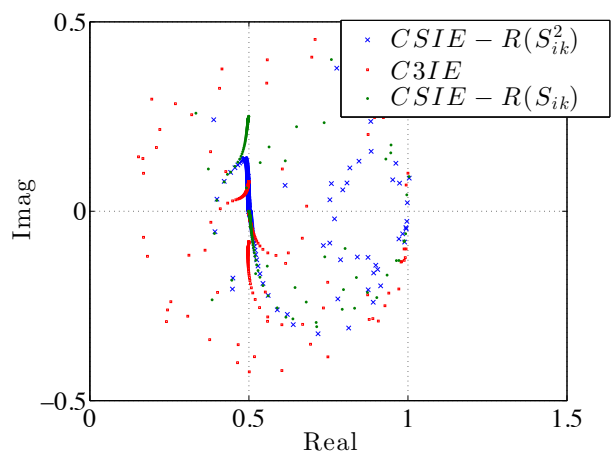

Fig. 2. Spectrum of two CSIE-AR methods and the C3IE for a unit sphere at $k=10$.
Figure 3 shows the singular values of the CSIE-AR with regularizing operator $\boldsymbol{n} \times S_{i k}^{2}$ at the same frequency. The limiting value of $1 / 2$ as $n \rightarrow \infty$ is (again) a reflection of the fact that the integral equation is of the second second kind. It is this property that prevents so-called "high density mesh breakdown".

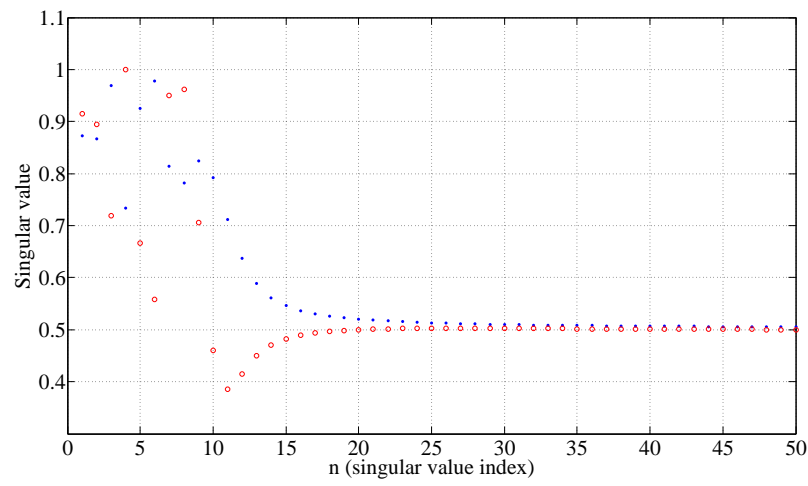

Fig. 3. Singular value decomposition of the $C S I E-S_{i k}^{2}$ for a unit sphere at $k=10$. Blue dots for coefficients of $\mathbf{U}_{\mathbf{n m}}$ and red dots for $\mathbf{X}_{n m}:=$ $\boldsymbol{n} \times \mathbf{U}_{n m}$

To summarize, the analytic behavior in the spherical case suggests that the proposed CSIE-AR approach will have good properties in terms of conditioning across the frequency spectrum and will be stable for dense and adaptive meshes.

\section{Discretization}

For the sake of simplicity, we assume the scatterer of interest has been modeled by a union of flat triangles. For discretization of each operator, we use zero order non-conformal piecewise constant basis functions and use collocation at the triangle centroid to define the system matrix. No charge continuity is enforced between triangles and no edge elements are introduced.

On each triangle we have one unknown for the fictitious scalar function $\sigma$ and two unknowns for the unknown current density (see figure 4) defined in terms of two orthogonal components of a local coordinate system [38].
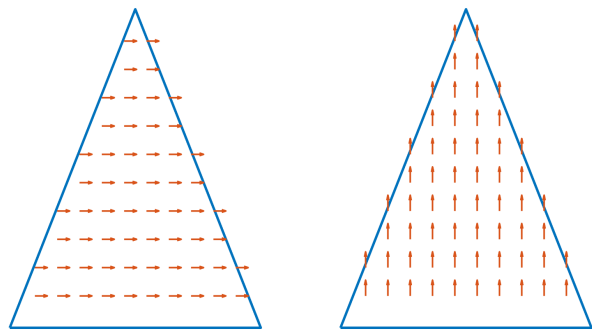

Fig. 4. Two orthogonal constant basis functions on each flat triangle for the unknown current.

An advantage of Fredholm equations of the second kind is that the resulting order of accuracy for the solution is 
simply the order of accuracy of the underlying quadrature rule [39], [40]. We solve the discrete linear system using GMRES iteration with FMM acceleration, resulting in a solution time of the order $O\left(n_{\text {iter }} N \log N\right)$ where $n_{\text {iter }}$ is the number of iterations required and $N$ is the number of unknowns. $n_{i t e r}$ depends on the conditioning of the system and its spectral properties [4], [1], [2], [3], [38].

Unlike the EFIE, the CFIE or the classical CSIE (which involve hypersingular operators), no preconditioning is required, instead we need to apply a regularizing operator which also requires the computation of operator products. Next we describe in more detail the discretization process for operator products. [12], [14].

For the calculation of the matrix elements of each integral operator we use standard quadrature rules for the far interactions and singularity subtraction for the self and near interactions. By doing this we can write each operator acting on a source density as a matrix-vector multiplication. For the discretization of each composition of operators, we use the same techniques applied on each operator independently (resulting in a standard matrix-matrix-vector multiplication). This will imply two standard FMM calls for a fast evaluation of the composition of two operators.

When the surface divergence of $\boldsymbol{J}$ is required, we make use of the following identity involving the regularizing operator $R$ :

$$
\begin{aligned}
\nabla_{\Gamma} \cdot \boldsymbol{J} & =\nabla_{\Gamma} \cdot \boldsymbol{n} \times S_{i k}^{2}[\boldsymbol{M}]=-\boldsymbol{n} \cdot \nabla \times S_{i k}^{2}[\boldsymbol{M}]= \\
& =-\boldsymbol{n} \cdot \nabla \times S_{i k}\left[S_{i k}[\boldsymbol{M}]\right]
\end{aligned}
$$

The discretization of the resulting composition of two operators $S_{i k}[\boldsymbol{M}]$ and $-\boldsymbol{n} \cdot \nabla \times S_{i k}[\cdot]$ is performed as mentioned in the first paragraph of this section (which implies two standard FMM calls, one to evaluate $S_{i k}[\boldsymbol{M}]$ and other to evaluate $-\boldsymbol{n} \cdot \nabla \times S_{i k}[\cdot]$ with a source given by the result of the first FMM call). Notice that the operator $-\boldsymbol{n} \cdot \nabla \times S_{i k}[\cdot]$ is a bounded operator, not hyper-singular (see [41] page 139).

Using the identity (30) and the vector identity $\nabla \times \nabla \times=$ $-\Delta+\nabla \nabla$, we can simplify the third term of the equation (28) in the following way:

$$
\begin{aligned}
& T\left[\boldsymbol{n} \times S_{i k}^{2}[\boldsymbol{M}]\right]=\boldsymbol{n} \times \nabla \times \nabla \times S_{k}\left[\boldsymbol{n} \times S_{i k}^{2}[\boldsymbol{M}]\right]= \\
& =\boldsymbol{n} \times k^{2} S_{k}\left[\boldsymbol{n} \times S_{i k}^{2}[\boldsymbol{M}]\right]+\boldsymbol{n} \times \nabla S_{k}\left[-\boldsymbol{n} \cdot \nabla \times S_{i k}^{2}[\boldsymbol{M}]\right]
\end{aligned}
$$

Notice that this will imply the composition of three integral operators, which are again discretized independently and therefore it will require three consecutive standard FMM calls.

We also use the identity $D_{i k}^{\prime} \cdot S_{i k}=S_{i k}^{\prime 2}-\frac{1}{4}$ to simplify the scalar part of equation (28) in the following way:

$$
\begin{aligned}
D_{k}^{\prime}\left[S_{i k}[\sigma]\right] & =\left(D_{k}^{\prime}-D_{i k}^{\prime}\right)\left[S_{i k}[\sigma]\right]+D_{i k}^{\prime}\left[S_{i k}[\sigma]\right] \\
& =\left(D_{k}^{\prime}-D_{i k}^{\prime}\right)\left[S_{i k}[\sigma]\right]+S_{i k}^{\prime 2}[\sigma]-\frac{\sigma}{4}
\end{aligned}
$$

where all the resulting operators are compact, and therefore, the singularity can be computed easily without facing any hypersingular kernel.

Here again, the composition of operators is computed in a similar way. The operator with difference kernel $D_{k}^{\prime}-D_{i k}^{\prime}$ is computed by doing two FMM calls for the far interaction and singularity substraction for the near interaction.

Notice that the proposed method implies the evaluation of compositions of integral operators, in that sense the proposed method is similar to the Calderon preconditioning technique. Nevertheless, we don't need to use special types of basis functions and test functions or barycentric meshes (see [13].)

\section{NUMERICAL EXAMPLES}

We show the properties of our method by testing first the absence of resonances, absence of low frequency breakdown and absence of high density mesh breakdown.

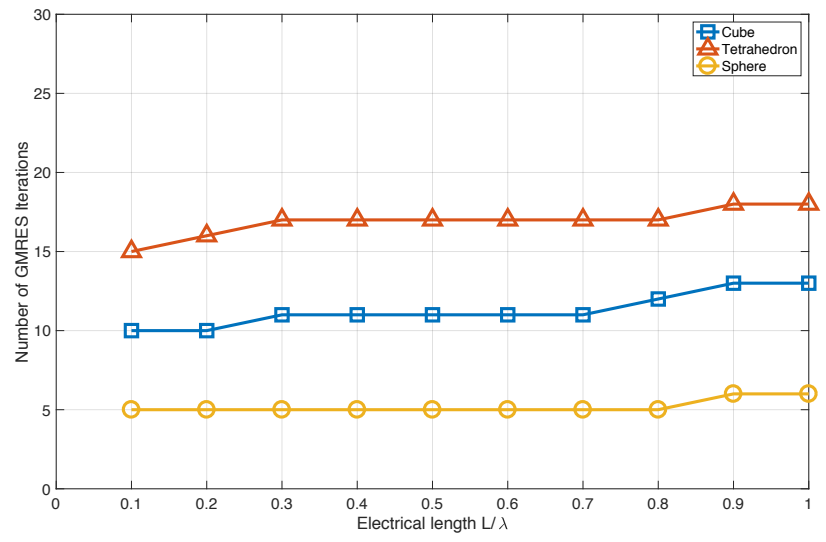

Fig. 5. Number of iterations vs electrical length of the scatterer for a cube of length $\mathrm{L}$, a tetrahedron of length $\mathrm{L}$ and a sphere of diameter $\mathrm{L}$.

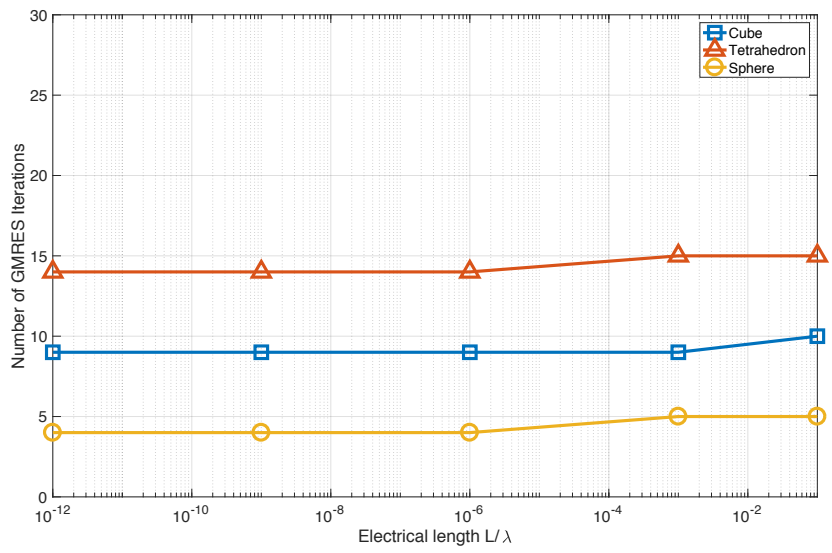

Fig. 6. Number of iterations vs electrical length of the scatterer for a cube of length $\mathrm{L}$, a tetrahedron of length $\mathrm{L}$ and a sphere of diameter $\mathrm{L}$. 


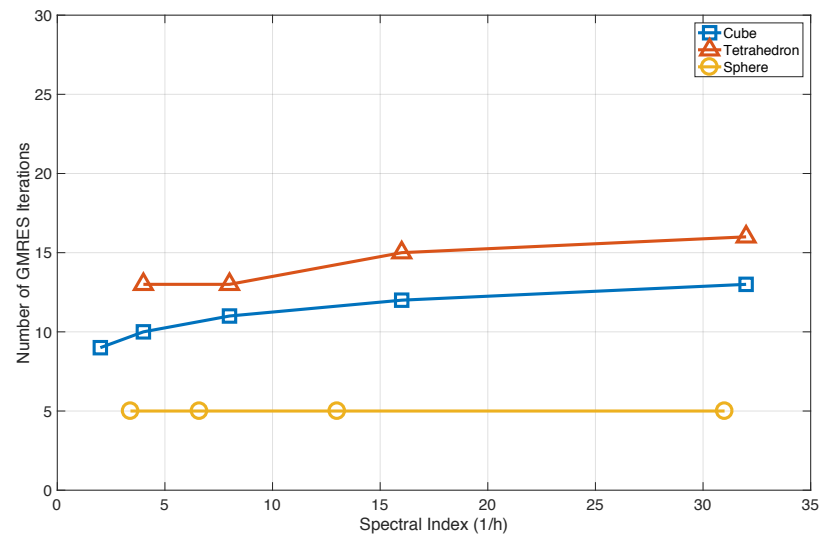

Fig. 7. Number of iterations vs spectral index for a cube of length $\mathrm{L}$, a tetrahedron of length $\mathrm{L}$ and a sphere of diameter $\mathrm{L}$.

Figure 5 shows that the number of iterations when running the iterative solver GMRES with FMM remains stable in a range between $L=0.1 \lambda$ and $L=1 \lambda$. No internal resonances are observed for a cubic, spheric and tetrahedral PEC scatterer.

Figure 6 shows that the number of iterations when running the iterative solver GMRES with FMM remains stable in a range between $L=0.1 \lambda$ and $L=10^{-12} \lambda$. No low frequency breakdown is observed for a cubic, spheric and tetrahedral PEC scatterer.

Figure 7 shows that the number of iterations when running the iterative solver GMRES with FMM remains stable in a range between $1 / h=3$ to $1 / h=33$. No high density mesh breakdown is observed for a cubic, spheric and tetrahedral PEC scatterer.

Next we illustrate the performance of our method with several benchmark geometries. All our calculations are carried out using an eight-core Intel Xeon E5-2680 at 2.7GHz. (Some rudimentary parallelization has been used, but the code has not been carefully optimized.)

For our first example, we consider the irregular decahedron shown in figure 8 (A) with a maximum dimension $D_{\max }=$ $0.5 \lambda$. We compare the result using the proposed equation $C S I E-S_{i k}^{2}$ with $C 3 I E$ and with a reference solution based on the Loop-Star RWG-CFIE using a much finer conformal mesh and a direct solver. As we can see from Figs. 10, 11, 12 , and 13 , we obtain better accuracy for the CSIE $-S_{i k}^{2}$ for both the electric and magnetic fields. The mesh used for the $C S I E-S_{i k}^{2}$ and $C 3 I E$ is non-conformal. In particular, the triangles in the upper half-space $(z \geq 0)$ and those in the lower half space ( $z \leq 0)$ that meet with edges along the $z=0$ plane are not aligned (see zoom in figure 9).
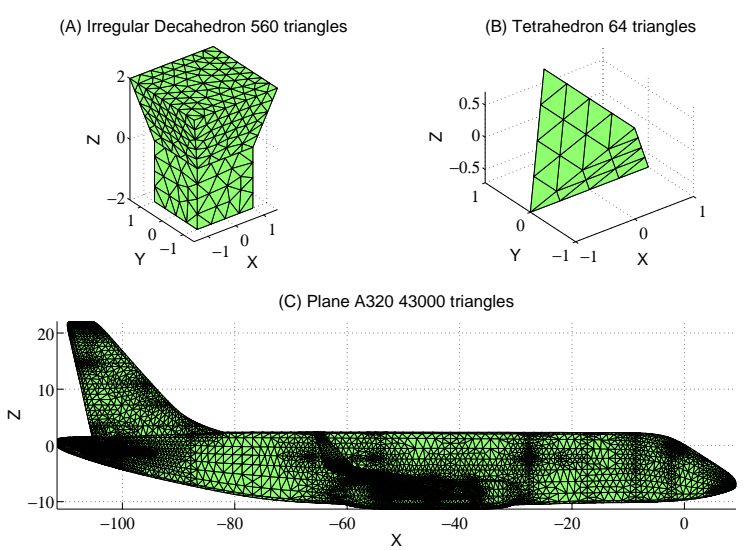

Fig. 8. Various testing geometries

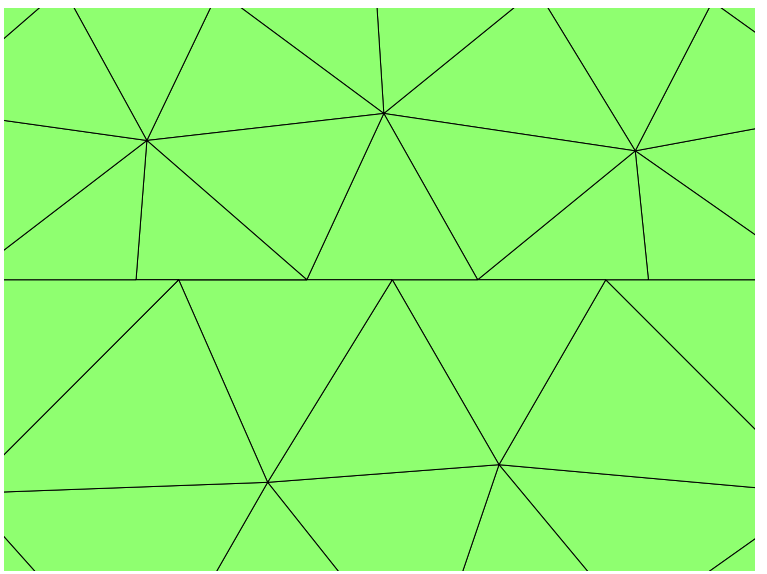

Fig. 9. Zoom of the non-conformal mesh in figure 8 (A).

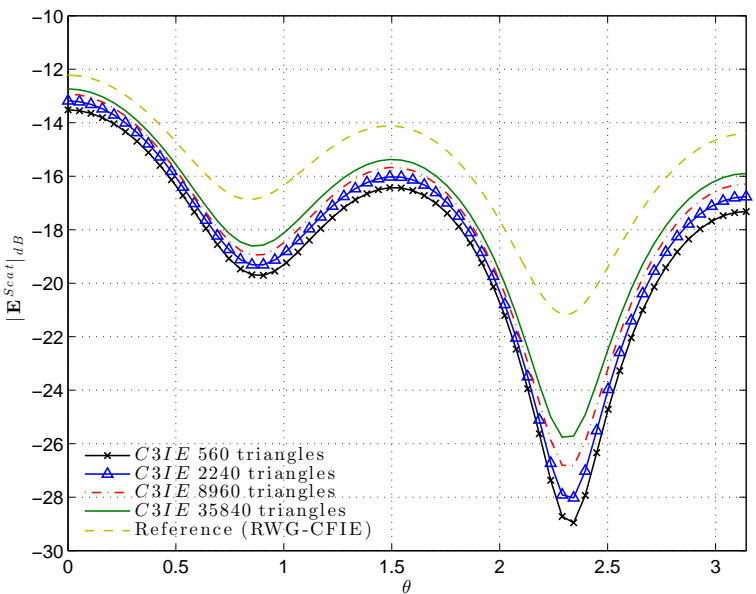

Fig. 10. The norm of the scattered electric field (in $\mathrm{dB}$ ) for a PEC decahedron of maximum dimension $D_{\max }=0.5 \lambda$. The target points are located at $R=$ $9 \mathrm{~m}$ and $\phi=0$ for an incoming plane wave $\boldsymbol{E}^{\text {in }}=\hat{x} \exp (i k z)$ of frequency $37.5 \mathrm{MHz}$. The solution is computed using the $C 3 I E$ formulation with FMM acceleration, a non-conformal mesh and zero order piecewise constant basis functions and collocation method. 
Our second example is a perfeclty conducting tetrahedron (figure 8 (B)), with a maximum dimension of $D_{\max }=0.5$. $10^{-12} \lambda$. We compare the result using the proposed equation $C S I E-S_{i k}^{2}$ with $C 3 I E$ and with a reference solution based on the Loop-Star RWG-CFIE using a much finer conformal mesh and a direct solver. As can be seen from Figs. 14 and 15 , we again obtain better accuracy with the $C S I E-S_{i k}^{2}$.

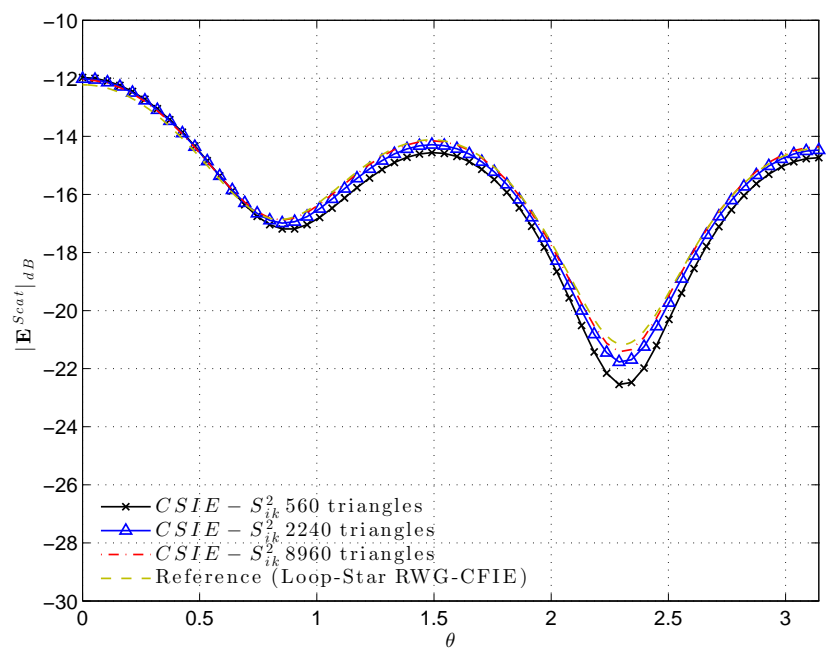

Fig. 11. The norm of the scattered electric field (in $\mathrm{dB}$ ) for a PEC decahedron of maximum dimension $D_{\max }=0.5 \lambda$. The target points are located at $R=9 \mathrm{~m}$ and $\phi=0$ for an incoming plane wave $\boldsymbol{E}^{\text {in }}=\hat{x} \exp (i k z)$ of frequency $37.5 \mathrm{MHz}$. The solution for the proposed CSIE- $S_{i k}^{2}$ formulation with FMM acceleration, a non-conformal mesh, and zero order piecewise constant basis functions and collocation method.

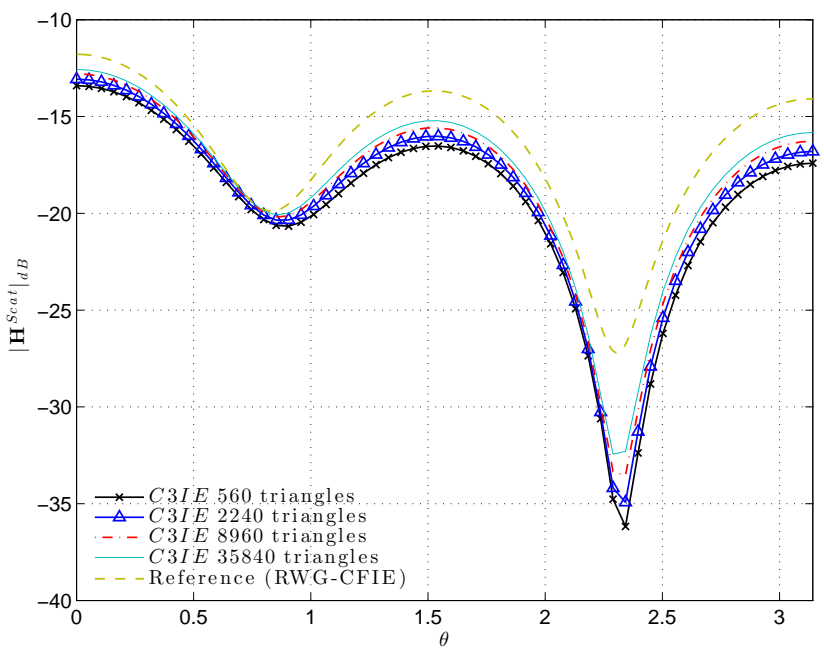

Fig. 12. The scattered magnetic field (in $\mathrm{dB}$ ) for a PEC decahedron of maximum dimension $D_{\max }=0.5 \lambda$. The target points are located at $R=9 \mathrm{~m}$ and $\phi=0$ for an incoming plane wave $\boldsymbol{E}^{\text {in }}=\hat{x} \exp (i k z)$ of frequency 37.5 MHz. The solution for the C3IE formulation with FMM acceleration, a non-conformal mesh, and zero order piecewise constant basis functions and collocation method.

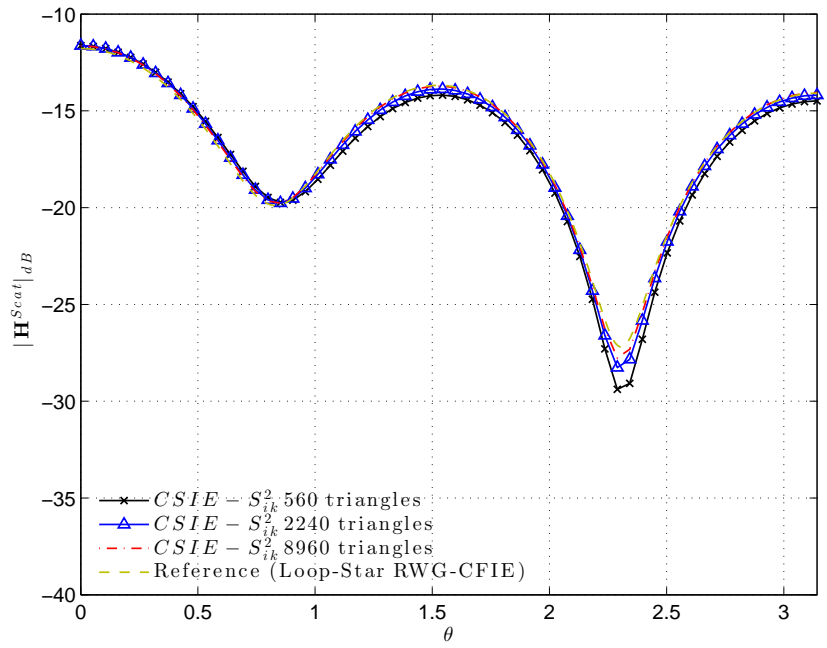

Fig. 13. The scattered magnetic field (in $\mathrm{dB}$ ) for a PEC decahedron of maximum dimension $D_{\max }=0.5 \lambda$. The target points are located at $R=9 \mathrm{~m}$ and $\phi=0$ for an incoming plane wave $\boldsymbol{E}^{\mathrm{in}}=\hat{x} \exp (i k z)$ of frequency 37.5 $\mathrm{MHz}$. The solution for the proposed CSIE $-S_{i k}^{2}$ formulation with FMM acceleration, a non-conformal mesh, and zero order piecewise constant basis functions and collocation method.

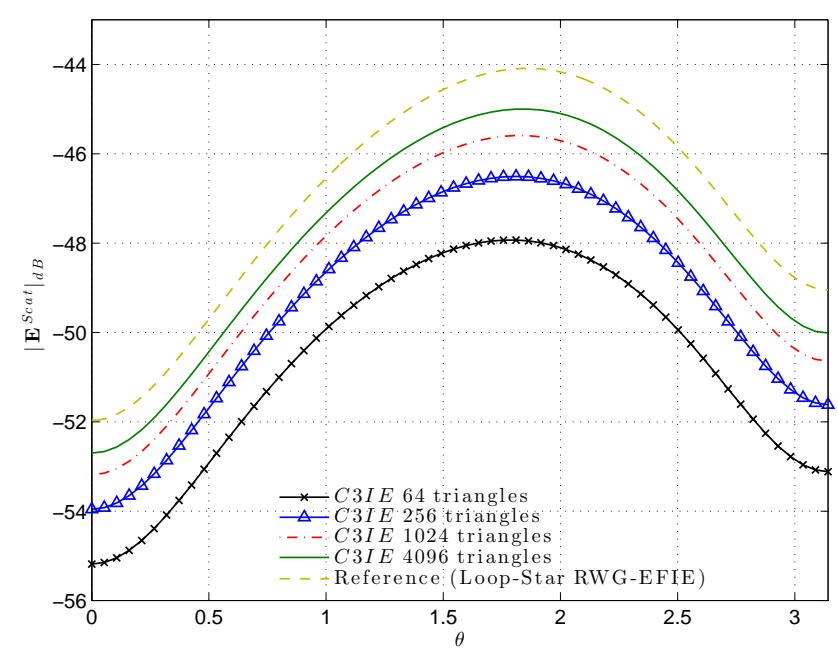

Fig. 14. The scattered electric field for a PEC tetrahedron of maximum dimension $D_{\max }=0.5 \cdot 10^{-12} \lambda$. The target points are located at $R=5 \mathrm{~m}$ and $\phi=0$ for an incoming plane wave $\boldsymbol{E}^{\text {in }}=\hat{x} \exp (i k z)$ of frequency $75 \mu \mathrm{Hz}$. The solution is computed using the $C 3 I E$ formulation with FMM acceleration, a non-conformal mesh, and zero order piecewise constant basis functions and collocation method. 


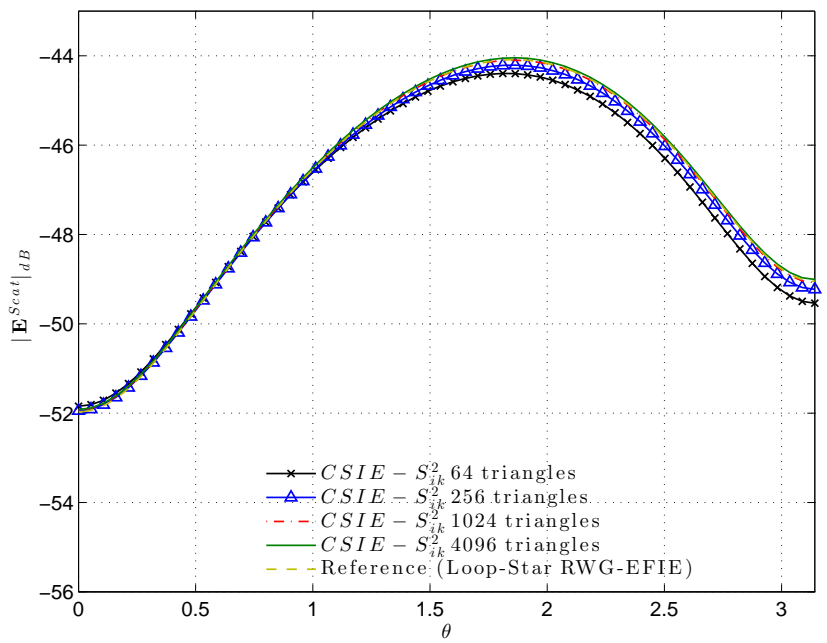

Fig. 15. The scattered electric field for a PEC tetrahedron of maximum dimension $D_{\max }=0.5 \cdot 10^{-12} \lambda$. The target points are located at $R=5 \mathrm{~m}$ and $\phi=0$ for an incoming plane wave $\boldsymbol{E}^{\text {in }}=\hat{x} \exp (i k z)$ of frequency $75 \mu \mathrm{Hz}$. The solution is computed using the CSIE $-S_{i k}^{2}$ formulation with FMM acceleration, a non-conformal mesh, and zero order piecewise constant basis functions and collocation method.

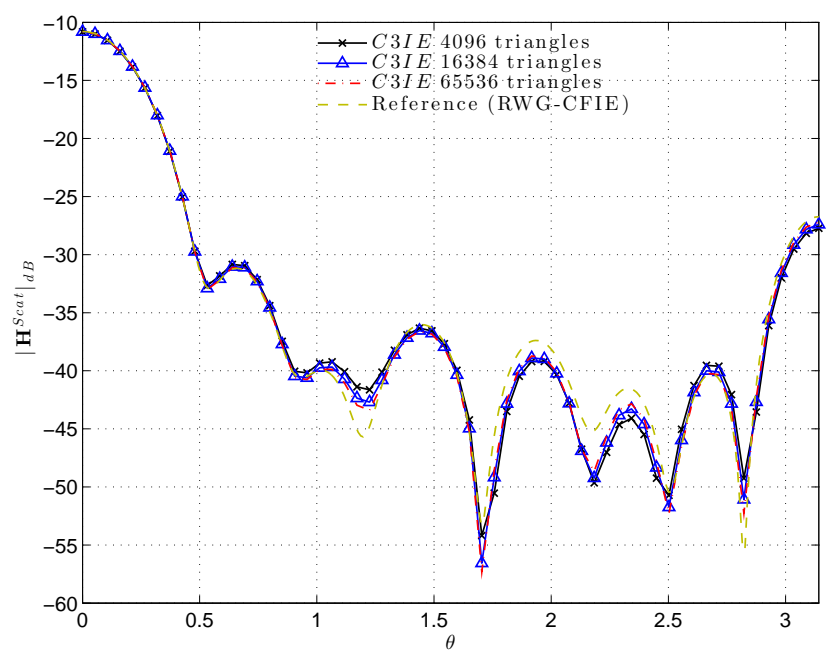

Fig. 16. The scattered electric field for a PEC tetrahedron of maximum dimension $D_{\max }=3 \lambda$. The target points are located at $R=10 \mathrm{~m}$ and $\phi=0$ for an incoming plane wave $\boldsymbol{E}^{\mathrm{in}}=\hat{x} \exp (i k z)$ of frequency $45 \mathrm{MHz}$. The solution is computed using the $C 3 I E$ formulation with FMM acceleration, a non-conformal mesh, and zero order piecewise constant basis functions and collocation method.

We next simulate scattering from the same geometry (the PEC tetrahedron) at a higher frequency, $D_{\max }=3 \lambda$. We obtain similar results in terms of accuracy (see Figs. 16,17).

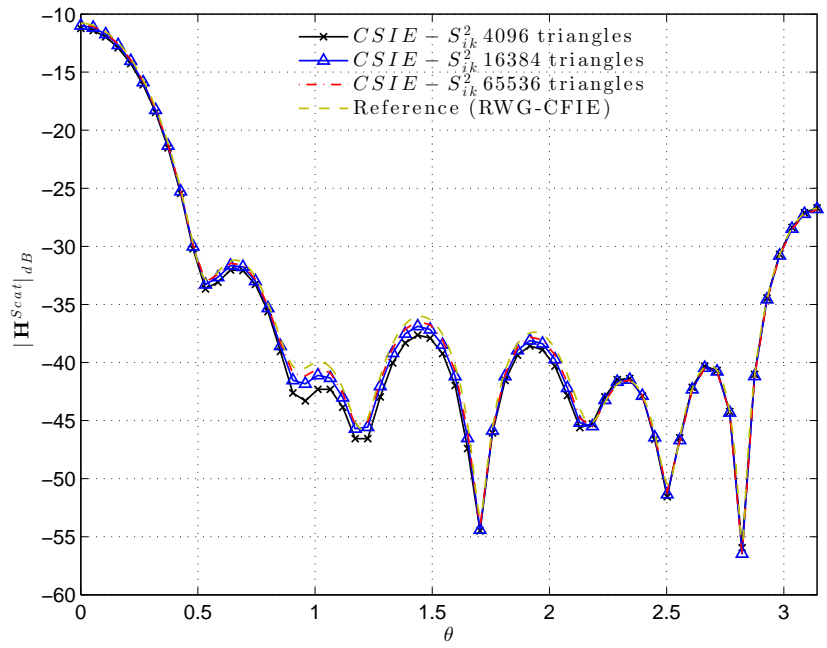

Fig. 17. The scattered electric field for a PEC tetrahedron of maximum dimension $D_{\max }=3 \lambda$. The target points are located at $R=10 \mathrm{~m}$ and $\phi=0$ for an incoming plane wave $\boldsymbol{E}^{\mathrm{in}}=\hat{x} \exp (i k z)$ of frequency $45 \mathrm{MHz}$. The solution is computed using the proposed $C S I E-S_{i k}^{2}$ formulation with FMM acceleration, a non-conformal mesh, and zero order piecewise constant basis functions and collocation method

For our last example, we consider a model of the A320 aircraft without engines (figure $8(\mathrm{C})$ ). The maximum dimension is $D_{\max }=3 \lambda$. We can see that the $C S I E-S_{i k}^{2}$ formulation gives consistent results for 43,000 and 122,000 triangles. (figure 18). To improve the condition number with irregular triangulation, we normalize each unknown by the square root of the triangle area, following the $L^{2}$ ideas proposed by Bremer [42]. Figure 18 shows the induced charge density $\sigma$ (the fictitious magnetic charge) on the aircraft surface.

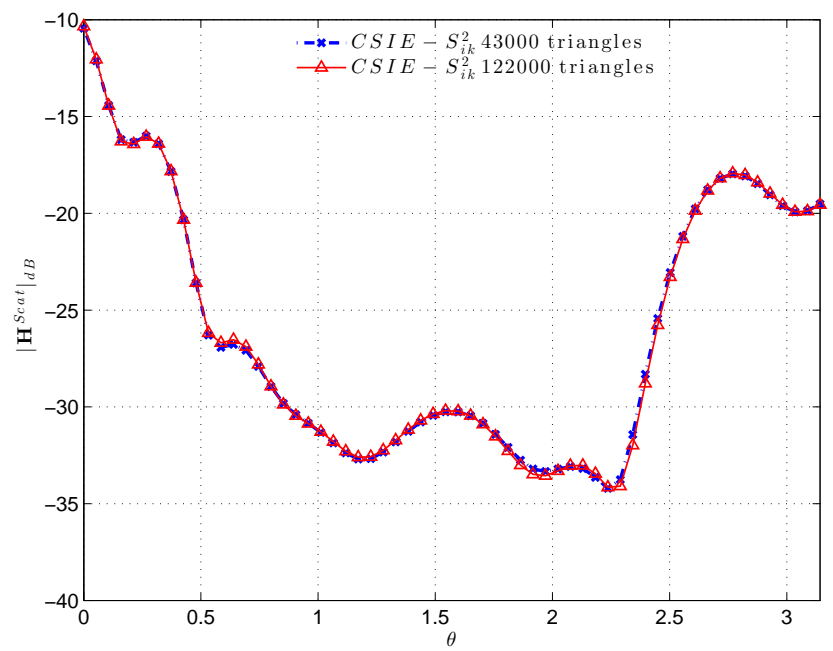

Fig. 18. The scattered electric field from a PEC aircraft of maximum dimension $D_{\max }=3 \lambda$. The target points are located at $R=25.5 \mathrm{~m}$ and $\phi=0$ for an incoming plane wave $\boldsymbol{E}^{\text {in }}=\hat{x} \exp (i k z)$ of frequency 26.5 $\mathrm{MHz}$. The solution is computed using the proposed $C S I E-S_{i k}^{2}$ formulation with FMM acceleration, a non-conformal mesh, and zero order piecewise constant basis functions and collocation method. 


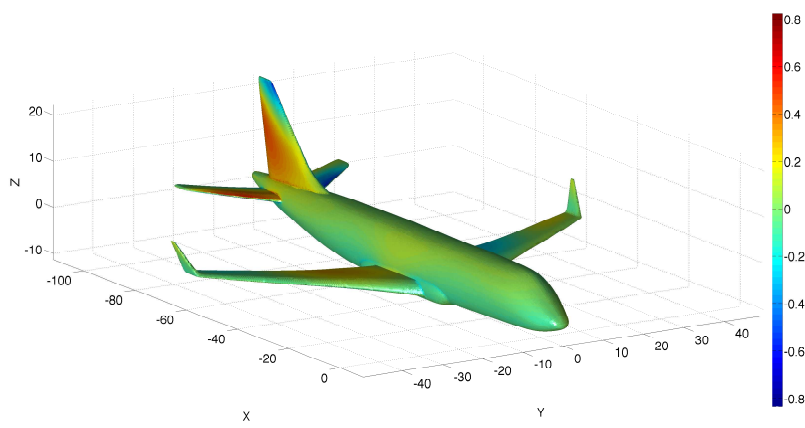

Fig. 19. Induced fictitious magnetic charge density $\sigma$ on a PEC aircraft of maximum dimension $D_{\max }=3 \lambda$ with an incoming plane wave $\boldsymbol{E}^{\text {in }}=$ $\hat{x} \exp (i k z)$ of frequency $26.5 \mathrm{MHz}$. The solution is computed using the proposed $C S I E-S_{i k}^{2}$ formulation with FMM acceleration, a non-conformal mesh, zero order piecewise constant basis functions and collocation method.

\section{CONCLUSIONS}

In this paper, we have presented an augmented, regularized combined source integral equation (CSIE-AR), which has useful properties across the frequency spectrum. In particular, it is resonance-free, is low frequency stable for simply connected geometries and immune from high density mesh breakdown. Unlike other second kind charge-current formulations, it performs well even for sharp geometries without substantial mesh refinement. The formulation is compatible with nonconformal meshes, which can be very useful when meshing complicated structures. Unlike hypersingular formulations, no preconditioning is required and approximate fast methods can be used without compromising accuracy. While RWG-based EFIE implementations are still more accurate in this low order, barely resolved regime, the proposed method is much easier to combine with high order basis functions and high order Nyström discretization (see [43], [44], [25]).

\section{ACKNOWLEDGMENT}

The authors would like to thank Zydrunas Gimbutas for many useful discussions and implementation help.

\section{REFERENCES}

[1] V. Rokhlin. Diagonal forms of translation operators for the helmholtz equation in three dimensions. Appl. Comput. Harmonic Anal., 1:82-93, 1993.

[2] W.C. Chew, E. Michielssen, J.M. Song, and J.M. Jin. Fast and efficient algorithms in computational electromagnetics. Artech House, Inc., 2001.

[3] H. Cheng, W.Y. Crutchfield, Z. Gimbutas, L. Greengard, J.F. Ethridge, J. Huang, V. Rokhlin, N. Yarvin, and J. Zhao. A wideband fast multipole method for the helmholtz equation in three dimensions. Journal of Computational Physics, 216(1):300-325, 2006.

[4] L. Greengard and V. Rokhlin. A fast algorithm for particle simulations. Journal of computational physics, 73(2):325-348, 1987.

[5] S. Rao, D. Wilton, and A. Glisson. Electromagnetic scattering by surfaces of arbitrary shape. Antennas and Propagation, IEEE Transactions on, 30(3):409-418, 1982.

[6] A.W. Maue. On the formulation of a general scattering problem by means of an integral equation. Z. Phys, 126(7):601-618, 1949.

[7] J.R. Mautz and R.F. Harrington. H-field, e-field, and combined field solutions for bodies of revolution. Technical report, SYRACUSE UNIV NY DEPT OF ELECTRICAL AND COMPUTER ENGINEERING, 1977.
[8] W. Wu, A.W. Glisson, and D. Kajfez. A study of two numerical solution procedures for the electric field integral equation at low frequency. Applied Computational Electromagnetics Society Journal, 10(3):69-80, 1995.

[9] G. Vecchi. Loop-star decomposition of basis functions in the discretization of the efie. Antennas and Propagation, IEEE Transactions on, 47(2):339-346, 1999.

[10] J. Zhao and W.C. Chew. Integral equation solution of maxwell's equations from zero frequency to microwave frequencies. Antennas and Propagation, IEEE Transactions on, 48(10):1635-1645, 2000.

[11] F.P. Andriulli, K. Cools, I. Bogaert, and E. Michielssen. On a well-conditioned electric field integral operator for multiply connected geometries. Antennas and Propagation, IEEE Transactions on, 61(4):2077-2087, 2013.

[12] K. Sertel and J.L. Volakis. Incomplete lu preconditioner for fmm implementation. Microwave and Optical Technology Letters, 26(4):265267, 2000.

[13] F.P. Andriulli, K. Cools, H. Bagci, F. Olyslager, A. Buffa, S. Christiansen, and E. Michielssen. A multiplicative calderon preconditioner for the electric field integral equation. Antennas and Propagation, IEEE Transactions on, 56(8):2398-2412, 2008.

[14] F.P. Andriulli and G. Vecchi. A helmholtz-stable fast solution of the electric field integral equation. Antennas and Propagation, IEEE Transactions on, 60(5):2357-2366, 2012.

[15] S.H. Christiansen and J.C. Nedelec. A preconditioner for the electric field integral equation based on Calderon formulas. SIAM J. Numer. Anal., 40(3):1100-1135, 2002.

[16] H. Contopanagos, B. Dembart, M. Epton, J.J Ottusch, V. Rokhlin, J.L. Visher, and S.M. Wandzura. Well-conditioned boundary integral equations for three-dimensional electromagnetic scattering. Antennas and Propagation, IEEE Transactions on, 50(12):1824-1830, 2002.

[17] D.L. Colton and R. Kress. Inverse acoustic and electromagnetic scattering theory, volume 93. Springer, 2013.

[18] S. Borel, D.P. Levadoux, and F. Alouges. A new well-conditioned integral formulation for maxwell equations in three dimensions. Antennas and Propagation, IEEE Transactions on, 53(9):2995-3004, 2005.

[19] O. Bruno, T. Elling, R. Paffenroth, and C. Turc. Electromagnetic integral equations requiring small numbers of krylov-subspace iterations. Journal of Computational Physics, 228(17):6169-6183, 2009.

[20] Y. Zhang, T.J. Cui, W.C. Chew, and J. Zhao. Magnetic field integral equation at very low frequencies. Antennas and Propagation, IEEE Transactions on, 51(8):1864-1871, 2003.

[21] M. Taskinen and P. Yla-Oijala. Current and charge integral equation formulation. Antennas and Propagation, IEEE Transactions on, 54(1):5867, 2006.

[22] M. Taskinen and D. Vanska. Current and charge integral equation formulations and picard's extended maxwell system. Antennas and Propagation, IEEE Transactions on, 55(12):3495-3503, 2007.

[23] A. Bendali, F. Collino, M. Fares, and B. Steif. Extension to nonconforming meshes of the combined current and charge integral equation. Antennas and Propagation, IEEE Transactions on, 60(10):4732-4744, Oct 2012.

[24] F. Vico, Z. Gimbutas, L. Greengard, and M. Ferrando-Bataller. Overcoming low-frequency breakdown of the magnetic field integral equation. IEEE transactions on antennas and propagation, 61(3):1285-1290, 2013.

[25] F. Vico, M. Ferrando-Bataller, A. Valero-Nogueira, and A. Berenguer. A high-order locally corrected nyström scheme for charge-current integral equations. Antennas and Propagation, IEEE Transactions on, 63(4):1678-1685, 2015.

[26] C. Epstein and L. Greengard. Debye sources and the numerical solution of the time harmonic maxwell equations. Communications on Pure and Applied Mathematics, 63(4):413-463, 2010.

[27] E. Chernokozhin and A. Boag. Method of generalized debye sources for the analysis of electromagnetic scattering by perfectly conducting bodies with piecewise smooth boundaries. Antennas and Propagation, IEEE Transactions on, 61(4):2108-2115, 2013.

[28] F. Vico, M. Ferrando, L. Greengard, and Z. Gimbutas. The decoupled potential integral equation for time-harmonic electromagnetic scattering. Communications on Pure and Applied Mathematics, 2015.

[29] Q.S. Liu, S. Sun, and W.C. Chew. A vector potential integral equation method for electromagnetic scattering. In Applied Computational Electromagnetics (ACES), 2015 31st International Review of Progress in, pages 1-2. IEEE, 2015.

[30] Classical Electrodynamics. JD Jackson. John Wiley \& Sons: New York, 1975. 
[31] C.H. Papas. Theory of electromagnetic wave propagation. Courier Dover Publications, 1988.

[32] Z. Qian and W.C. Chew. Fast full-wave surface integral equation solver for multiscale structure modeling. IEEE Transactions on Antennas and Propagation, 57(11):3594-3601, 2009.

[33] M. Taskinen P. Yla-Oijala and S. Jarvenpaa. Advanced surface integral equation methods in computational electromagnetics. In Electromagnetics in Advanced Applications, 2009. ICEAA'09. International Conference on, pages 369-372. IEEE, 2009.

[34] W. Bruno, T. Elling, and C. Turc. Well-conditioned high-order algorithms for the solution of three-dimensional surface acoustic scattering problems with neumann boundary conditions. J. Numer. Meth. Eng, 91(10), 2009.

[35] R. Kress. Minimizing the condition number of boundary integral operators in acoustic and electromagnetic scattering. The Quarterly Journal of Mechanics and Applied Mathematics, 38(2):323-341, 1985.

[36] F. Vico, Z. Gimbutas, and L. Greengard. Boundary integral equation analysis on the sphere. Numer. Math., 128:463-487, 2014.

[37] G.C. Hsiao and R.E. Kleinman. Mathematical foundations for error estimation in numerical solutions of integral equations in electromagnetics. Antennas and Propagation, IEEE Transactions on, 45(3):316-328, 1997.

[38] Z. Gimbutas and L. Greengard. Simple fmm libraries for electrostatics, slow viscous flow, and frequency-domain wave propagation. Commun. Comput. Phys., 18:516-528, 2015.

[39] K.E. Atkinson. A survey of numerical methods for the solution of Fredholm integral equations of the second kind. Number v. 12 in Miscellaneous Bks. Society for Industrial and Applied Mathematics, 1976.

[40] PM Anselone. Uniform approximation theory for integral equations with discontinuous kernels. SIAM Journal on Numerical Analysis, 4(2):245253, 1967.

[41] D.L. Colton and R. Kress. Integral equation methods in scattering theory, volume 57. Wiley New York, 1983.

[42] J. Bremer. On the Nyström discretization of integral equations on planar curves with corners. Appl. Comput. Harmonic Anal., 32:45-64, 2012.

[43] A. Klöckner, A. Barnett, L. Greengarde, and M. ONeil. Quadrature by expansion: A new method for the evaluation of layer potentials. Journal of Computational Physics, 252:332-349, 2013.

[44] C.L. Epstein, L. Greengard, and A. Klockner. On the convergence of local expansions of layer potentials. SIAM Journal on Numerical Analysis, 51(5):2660-2679, 2013.

Felipe Vico was born in Valencia, Spain, in 1981. He received the M.S degree in 2004 in Telecommunication engineering from the Polytechnic University of Valencia and the Ph.D. degree in 2009 in Telecommunications engineering also from UPV. He received the M.S in Mathematics in 2009 from Universidad Nacional de Educacion a Distancia. He received the best paper award in EUCAP 2006. During 2010 he stayed for several months as a guest researcher at the Courant Institute of Mathematical Sciences, in New York City, USA $\mathrm{He}$ is now assistant Professor in Universidad Politcnica de Valencia. His research interests are Numerical methods for CEM, high frequency methods and integral equation methods.

Leslie Greengard (M'07) was born in London, England on April 19, 1957. He received a B.A. degree in Mathematics from Wesleyan University in 1979, a Ph.D. degree in Computer Science from Yale University in 1987 , and an M.D. degree from Yale University in 1987. From 1987-1989 he was an NSF Postdoctoral Fellow at Yale University and at the Courant Institute of Mathematical Sciences, NYU, where he has been a faculty member since 1989. He was the Director of the Courant Institute from 2006-2011 and is currently Director of the Simons Center for Data Analysis at the Simons Foundation in New York, NY. His research interests include fast algorithms, computational acoustics and electromagnetics, elasticity, heat transfer, fluid dynamics, and medical imaging. Prof. Greengard is a member of the National Academy of Sciences and the National Academy of Engineering.
Miguel Ferrando-Bataller (S81M83) was born in Alcoy, Spain, in 1954 $\mathrm{He}$ received the M.S. and Ph.D. degrees in telecommunication engineering from the Universidad Politecnica de Catalunya, Barcelona, Spain, in 1977 and 1982, respectively. From 1977 to 1982, he was a Teaching Assistant with the Antennas, Microwave, and Radar Group, Universidad Politcnica de Catalunya, and in 1982 he became an Associate Professor. In 1990, he joined the Universidad Politecnica de Valencia, Valencia, Spain, where he was Director of the Telecommunication Engineering School and Vice-Chancellor. He was Director of Long-life learning Office and he is currently Professor of antennas and satellite communications. His current research interest includes numerical methods, antenna design and e-learning activities.

Eva Antonino-Daviu was born in Valencia, Spain, on July 10, 1978. She received the M.S. and Ph.D. degrees in electrical engineering from the Universidad Politcnica de Valencia, Valencia, Spain, in 2002 and 2008, respectively. In 2002, she joined the Electromagnetic Radiation Group, Universidad Politcnica de Valencia, and in 2005 she became a Lecturer at the Escuela Politcnia Superior de Gandia, Gandia, Spain. During 2005 she stayed for several months as a guest researcher at the Department of Antennas and EM Modelling of IMST GmbH, in Kamp-Lintfort, Germany. Eva AntoninoDaviu has published over 50 papers in international technical journals and renowned conferences in the field of antennas and propagation, and she is a regular lecturer in European School of Antennas courses. Her current research interests include wideband and multi-band planar antenna design, modal analysis of antennas, MIMO and antenna design for mmwave applications. Eva Antonino-Daviu was awarded the Premio Extraordinario de Tesis Doctoral from the Universidad Politenica de Valencia in 2008 